\title{
Decrypting the warped black strings
}

\author{
Monica Guica \\ David Rittenhouse Laboratory, University of Pennsylvania, \\ Philadelphia, PA 19104-6396, USA
}

\begin{abstract}
:
We propose a map for extracting the holographic meaning of the metric for a class of warped $\mathrm{AdS}_{3}$ black strings in type IIB supergravity. Our choice of holographic data is based upon a general prescription due to Papadimitriou. With this choice, we find a holographic stress tensor that is both symmetric and conserved, at least when restricted to a particular sector of the theory. Using the holographic stress tensor, we compute the energy and momentum per unit length of the black strings, as well as the conformal anomaly, and we show that the results agree with those previously obtained via covariant methods.
\end{abstract}




\section{Contents}

1. Introduction

2. Setup and review 2

3. Dipole CFTs at finite temperature 6

4. Holography 8

4.1 The finite-temperature FG expansion 8

4.2 Holographic renormalization 10

4.3 The holographic stress tensor 14

4.4 General warped black strings 15

5. Discussion 16

\section{Introduction}

Holography is believed to be a fundamental property of quantum gravity [1]; nevertheless, the holographic dictionary has only been understood in detail for the case of the AdS/CFT correspondence [2-7] and a few other, qualitatively similar, cases. Examples include Lifshitz spacetimes [8-12], non-conformal branes [13,14], non-AdS/QCD [15-18], etc.

It is interesting to inquire whether there exist any tractable examples where the holographic dictionary is not just a straightforward extension of the AdS/CFT one. It appears that Schrödinger spacetimes [19], which geometrically realize the non-relativistic conformal group, provide such an example. Aside from their theoretical appeal, Schrödinger spacetimes also have many potentially interesting applications, for example as gravity duals to strongly-coupled non-relativistic conformal field theories $[19,20]$ and, in the threedimensional case ${ }^{1}$, as toy models for the Kerr/CFT correspondence [21,22].

The main challenge in understanding holography for Schrödinger spacetimes is how to correctly identify the holographic sources to which boundary operators - and in particular the dual stress tensor - couple. In a beautiful paper [23], Papadimitriou gave a general prescription for choosing the holographic data in an arbitrary spacetime, at least at the classical level. In [24] this prescription was applied to study pure gauge modes in threedimensional Schrödinger spacetimes and relate them to sources and expectation values for the putative holographic stress tensor. Although the dual theory is believed to be nonlocal and non-relativistic [25-28], the holographic stress tensor uncovered in [24] is both symmetric and conserved, signaling the presence of an emergent relativistic symmetry.

\footnotetext{
${ }^{1}$ Three-dimensional Schrödinger spacetimes are also known as null warped $\mathrm{AdS}_{3}$. Their finitetemperature version, which is the subject of this paper, is called (spacelike) warped $\mathrm{AdS}_{3}$.
} 
Hints of a relativistic conformal symmetry $[29,30]$ have long been present in the study of warped $\mathrm{AdS}_{3}$ black hole/string backgrounds, which in the context of string theory correspond to finite-temperature states in three-dimensional Schrödinger spacetimes. Asymptotic symmetry group analyses of these spacetimes reveal properties akin to those of twodimensional relativistic conformal field theories, such as the presence of two (mutually exclusive) Virasoro algebras and the applicability of Cardy's formula for the entropy $[31,32]$.

In this note, we propose an extension of the dictionary in [24] to a family of warped $\mathrm{AdS}_{3}$ black string solutions in string theory found in [32]. As in the pure Schrödinger case, the holographic stress tensor we find is both symmetric and conserved, in accordance with the results of the asymptotic symmetry group analyses. We also show that the expectation value of the holographic stress tensor and the conformal anomaly in the warped black string backgrounds agree with the energy and momentum of the strings, as well as the conformal anomaly computed via other means in $[31,32]$. This match brings further support in favour of the holographic dictionary we are proposing.

Our dictionary, which is based on Papadimitriou's prescription, is not the only dictionary that has been put forth for understanding the holographic stress tensor in Schrödinger spacetimes. Other attempts include [11,33], of which [33] has carried out an extremely careful and detailed analysis. These prescriptions are different from ours in that they choose different holographic sources, which results in different operator expectation values and different holographic Ward identities. While the tests that our proposal passes are certainly encouraging, they only involve matching a gravity calculation to another gravity calculation. Ultimately, one should use input from the dual field theory in order to decide on the correct holographic data and the Ward identities they satisfy ${ }^{2}$.

This paper is organised as follows. In section 2, we review the holographic proposal of [24] for three-dimensional Schrödinger spacetimes, as well as the simplest warped black string solution of [32]. In section 3, we discuss the modifications of this proposal that are necessary for applying it to the warped black string backgrounds. In section 4 , we construct a truncated holographic dictionary and compute the expectation value of the stress tensor for all the black string backgrounds of [32]. We conclude with a discussion in section 5 .

\section{Setup and review}

We start from $A d S_{3}$ in Poincaré coordinates, with metric

$$
\frac{d s^{2}}{\ell^{2}}=\frac{d w^{+} d w^{-}+d z^{2}}{z^{2}}
$$

This spacetime has $S L(2, \mathbb{R})_{L} \times S L(2, \mathbb{R})_{R}$ isometry. The $S L(2, \mathbb{R})_{R}$ generators are given by (minus) $H_{i}$, where

\footnotetext{
${ }^{2}$ In certain cases, the dual field theory to Schrödinger spacetimes is known: it is a so-called null dipole theory [34-36] or an RG flow thereof. Dipole theories are similar to non-commutative ones, so it may be possible to find - perhaps using the methods of $[37,38]$ - the gauge-invariant operator that couples to the spacetime metric.
} 


$$
H_{-1}=\partial_{+}, \quad H_{0}=w^{+} \partial_{+}+\frac{1}{2} z \partial_{z}, \quad H_{1}=\left(w^{+}\right)^{2} \partial_{+}+w^{+} z \partial_{z}-z^{2} \partial_{-}
$$

and the same expressions, with $w^{+} \leftrightarrow w^{-}$, yield the $S L(2, \mathbb{R})_{L}$ ones. Lowering the index on the above Killing vectors, we find the left-invariant one-forms

$$
\sigma_{-1}=\frac{d w^{-}}{z^{2}}, \quad \sigma_{0}=\frac{w^{+} d w^{-}}{z^{2}}+\frac{d z}{z}, \quad \sigma_{1}=\left(w^{+}\right)^{2} \frac{d w^{-}}{z^{2}}+2 w^{+} \frac{d z}{z}-d w^{+}
$$

These one-forms are all invariant under $S L(2, \mathbb{R})_{L} \times U(1)_{R}$, where for each of the forms $\sigma_{i}$, the $U(1)_{R}$ is generated by the Killing vector $H_{i}$ and is non-compact.

\section{$\diamond$ Three-dimensional Schrödinger spacetimes}

A three-dimensional Schrödinger spacetime is obtained by deforming $A d S_{3}$ by the one-form $\sigma_{-1}$ dual to the null Killing vector field $H_{-1}=\partial_{+}$

$$
\frac{d s^{2}}{\ell^{2}}=\frac{d w^{+} d w^{-}+d z^{2}}{z^{2}}-\frac{\lambda^{2}\left(d w^{-}\right)^{2}}{z^{4}}
$$

By construction, the above spacetime has $S L(2, \mathbb{R})_{L} \times U(1)_{R}$ isometry, where the $U(1)_{R}$ corresponds to translations along $w^{+}$. Schrödinger spacetimes are not solutions of Einstein gravity alone, one needs to add matter to support them. In many cases, the matter can be modeled by a (topologically) massive vector field

$$
A=\lambda^{\prime} \ell \sigma_{-1}=\frac{\lambda^{\prime} \ell d w^{-}}{z^{2}}
$$

The relationship between $\lambda$ and $\lambda^{\prime}$ is fixed by the equations of motion. Any scalars that may be present in the action must be constant in a Schrödinger background, due to the $S L(2, \mathbb{R})_{L}$ symmetry.

\section{$\diamond$ Field theory duals}

In general, the field theory duals to three-dimensional Schrödinger spacetimes are not known. Nevertheless, in [39] it was argued that, at least at large $N$ and strong coupling, they can be effectively described as a finite deformation of a two-dimensional CFT by a $(1,2)$ primary operator. This operator, although irrelevant from the point of view of the usual conformal group, is however exactly marginal with respect to the $S L(2, \mathbb{R})_{L}$ conformal symmetry

$$
S=S_{C F T}+\lambda \int d^{2} w \mathcal{O}_{(1,2)}
$$

The above equation provides a formal definition of the field theory duals to three-dimensional Schrödinger spacetimes, in the given region of parameter space. In the following, we will denote the theories defined via $(2.6)$ as dipole $C F T s^{3}$.

\footnotetext{
${ }^{3}$ This terminology was used in [31] to denote the two-dimensional field theory dual to a particular three-dimensional Schrödinger spacetime in string theory, which is described by the low-energy limit of the dipole-deformed [34] D1-D5 gauge theory. Since we expect that all field theories dual to three-dimensional Schrödinger spacetimes share a certain number of universal characteristics, herein we will denote all of them as dipole CFTs.
} 


\section{$\diamond$ Linearized holographic data}

The first step in understanding any holographic dictionary [7] is to find the most general solution to the asymptotic equations of motion and isolate its normalizable and non-normalizable components. At linearized level, all perturbations of three-dimensional Schrödinger spacetimes have been classified [24,39]. They consist of pure gauge modes (that can be induced by a diffeomorphism), which we will call T-modes, and "true" propagating degrees of freedom, which we call X-modes. Both types of modes are present in the linearized solution for the metric and the massive vector

$$
\delta g_{i j}=\delta g_{i j}^{T}+\delta g_{i j}^{X}, \quad \delta A_{\mu}=\delta A_{\mu}^{T}+\delta A_{\mu}^{X}
$$

Unfortunately, there is no obvious choice of holographic sources among the various functions of $w^{ \pm}$that parametrize the above solutions, leading to the different proposals in the literature. In the following, we review a general prescription due to Papadimitriou [23] that can determine the holographic sources in an arbitrary spacetime with a timelike boundary.

\section{$\diamond$ Papadimitriou's prescription}

The proposal of [23] is based on the observation that holographic renormalization can be viewed as a canonical transformation that renders the variational principle at the asymptotic space-time boundary well-defined. The role of time is played by the radial coordinate $z$. This canonical transformation diagonalizes the map between the cotangent bundle of the phase space, parametrized by $\left(d q^{I}, d p_{I}\right)$, and that of the space of asymptotic solutions, parametrized by $\left(d \alpha^{I}, d \beta_{I}\right)$. Thus, holographic renormalization typically corresponds to

$$
\left(\begin{array}{c}
q^{I} \\
p_{I}
\end{array}\right) \underset{\text { transformation }}{\stackrel{\text { canonical }}{\longrightarrow}}\left(\begin{array}{c}
\tilde{q}^{I} \\
\tilde{p}_{I}
\end{array}\right) \sim\left(\begin{array}{cc}
z^{-2 \nu} & 0 \\
0 & z^{2 \nu}
\end{array}\right)\left(\begin{array}{c}
\alpha^{I} \\
\beta_{I}
\end{array}\right)
$$

for some $\nu>0$. Since this transformation is canonical, the symplectic form is unchanged

$$
\Omega=d q^{I} \wedge d p_{I}=d \tilde{q}^{I} \wedge d \tilde{p}_{I}=d \alpha^{I} \wedge d \beta_{I}
$$

In this framework, finding the holographic sources and expectation values is straightforward: one simply needs to evaluate the symplectic form on a general solution to the asymptotic equations of motion and then diagonalize it. The resulting diagonal entries are the holographic sources and expectation values.

It is not hard to show [24] that the symplectic form for linearized modes in threedimensional Schrödinger spacetimes takes the diagonal form

$$
\Omega \sim \int T \wedge T+X \wedge X
$$

and moreover the source for the T-modes is a two-dimensional symmetric tensor $\delta g_{i j}^{(0)}$. Applying the prescription above, we conclude that the $\mathrm{X}$ and the $\mathrm{T}$ modes couple to different operators in the dual field theory, and in particular the $\mathrm{T}$ modes source the dual stress tensor. 


\section{$\diamond$ A Fefferman-Graham-like expansion}

If one is interested solely in the holographic stress tensor, it is possible to consistently set the X-modes to zero. In this case, it was shown in [24] that the general non-linear solution for the T-modes is given by

$$
g_{\mu \nu}^{T}=\hat{g}_{\mu \nu}-A_{\mu}^{T} A_{\nu}^{T}
$$

where $\hat{g}_{\mu \nu}$ is the metric of an auxiliary $A d S_{3}$ spacetime of radius $\ell$ and takes the general Fefferman-Graham form. The vector field $A_{\mu}^{T}$ solves

$$
F_{\mu \nu}=\frac{2}{\ell} \hat{\epsilon}_{\mu \nu \rho} A^{\rho}, \quad A_{\mu} \hat{A}^{\mu}=0
$$

The symplectic form evaluated on this solution is identical ${ }^{4}$ to that of the auxiliary $\mathrm{AdS}_{3}$ spacetime. By Papadimitriou's prescription, we choose the holographic sources to be those of the auxiliary $\mathrm{AdS}_{3}$. This choice leads to a holographic stress tensor that is symmetric, conserved and yields the same conformal anomaly as the $A d S_{3}$ one, at least when evaluated on the T-modes only.

\section{$\diamond$ The warped black strings}

The outcome of the analysis of [24] is rather intriguing and it would be useful to test its predictions in cases where an independent computation is possible. Black holes/strings provide a good testing ground, as one can use covariant methods [41] to compute various non-trivial conserved charges and anomalies, which can then be compared with predictions from the holographic stress tensor.

The simplest theories of gravity coupled to massive vector fields do not seem to contain generic enough (non-extremal) black hole solutions. Nevertheless, such solutions do abound in string theory. In [32], a four-parameter family of stringy warped analogues of the nonextremal BTZ black string (carrying both mass and momentum) has been constructed. The simplest warped black string in this family can be viewed as a solution of the following three-dimensional action

$$
S=\frac{1}{16 \pi G_{3}} \int d^{3} x \sqrt{g}\left(R-4(\partial U)^{2}-\frac{4}{\ell^{2}} e^{-4 U} A^{2}+\frac{2}{\ell^{2}} e^{-4 U}\left(2-e^{-4 U}\right)-\frac{1}{\ell} \epsilon^{i j k} A_{i} F_{j k}\right)
$$

which descends from a consistent truncation of type IIB supergravity [32]. The warped black string solution is

$$
\begin{gathered}
\frac{d s_{3}^{2}}{\ell^{2}}=T_{+}^{2} d x_{+}^{2}+\left[T_{-}^{2}\left(1+\tilde{\lambda}^{2} T_{+}^{2}\right)-\tilde{\lambda}^{2} r^{2}\right] d x_{-}^{2}+2 r d x^{+} d x^{-}+\frac{\ell^{2} d r^{2}}{4\left(r^{2}-T_{+}^{2} T_{-}^{2}\right)}\left(1+\tilde{\lambda}^{2} T_{+}^{2}\right) \\
A=\frac{\tilde{\lambda} \ell}{\sqrt{1+\tilde{\lambda}^{2} T_{+}^{2}}}\left(r d x^{-}+T_{+}^{2} d x^{+}\right), \quad e^{4 U}=1+\tilde{\lambda}^{2} T_{+}^{2}
\end{gathered}
$$

\footnotetext{
${ }^{4}$ Provided we use radial gauge in the Schrödinger spacetime. Explicit linearized calculations show that the symplectic form is not conserved with respect to the radial coordinate of the auxiliary $\mathrm{AdS}_{3}$, but it is conserved with respect to the Schrödinger radial coordinate.
} 
The parameters $T_{ \pm}$are related to the Hawking temperature and horizon velocity via

$$
T_{H}=\frac{2}{\pi} \frac{T_{+} T_{-}}{T_{+}+T_{-}}, \quad \Omega_{H}=\frac{T_{+}-T_{-}}{T_{+}+T_{-}}
$$

In [32] it was shown that the energy and momentum per unit length of these black strings are given by

$$
E \pm P=\frac{\ell}{4 \pi G_{3}} T_{ \pm}^{2}
$$

Moreover, asymptotic symmetry group analyses indicate the presence of either a leftmoving or a right-moving Virasoro symmetry with central charge

$$
c=\frac{3 \ell}{2 G_{3}}
$$

These properties can be shown to hold with minor modifications also for the general fourparameter family of warped black strings [32]. We will come back to these more general solutions in section 4.4 .

\section{$\diamond$ Objectives}

We would like to extract from the metric (2.14) which operator sources and expectation values have been turned on, and to reproduce $E, P$ and $c$ above from a holographic stress tensor computation, as has been done in [44-46] for the case of AdS. The challenge is that the black hole spacetimes are not diffeomorphic to the vacuum Schrödinger background, so the T-mode analysis in [24] does not strictly apply. In this paper we propose a generalization of [24] that reproduces the above quantities from a holographic computation.

\section{Dipole CFTs at finite temperature}

Any two-dimensional theory can be put at finite temperature ${ }^{5}$ by restricting the space where it is defined to a Rindler wedge of Minkowski space. The Rindler coordinates $x^{ \pm}$ are related to the Minkowski coordinates $w^{ \pm}$via the coordinate transformation $w^{ \pm}=$ $e^{2 T_{ \pm} x^{ \pm}}$. In a two-dimensional conformal field theory, this is a conformal transformation which induces a thermal expectation value for the stress tensor.

A natural way to extend the definition (2.6) of dipole CFTs to Rindler space is

$$
S=S_{C F T}+\lambda \int d x^{+} d x^{-} \mathcal{O}_{(1,2)}\left(x^{+}, x^{-}\right)
$$

which is the only $(1,2)$ deformation that is consistent with translation invariance in the $x^{ \pm}$plane. We are assuming that $\lambda$ is $T_{+}$-independent. If we map the deformation back to Minkowski space, we find

$$
\lambda \int d x^{+} d x^{-} \mathcal{O}_{(1,2)}\left(x^{+}, x^{-}\right) \rightarrow 2 \lambda T_{+} \int d w^{+} d w^{-} w^{+} \mathcal{O}_{(1,2)}\left(w^{+}, w^{-}\right)
$$

\footnotetext{
${ }^{5}$ We will be interested in the more general setup where we turn on both a temperature $T_{H}$ and a potential conjugate to momentum $\Omega_{H}$, whose relationships to $T_{ \pm}$are given in (2.15). The parameters $T_{ \pm}$are related to the usual left/right moving temperatures defined in e.g. [47] via $T_{ \pm}=\pi T_{R / L}$.
} 
where we used the fact that $\mathcal{O}_{(1,2)}$ is a conformal primary. The deformation on the right hand side still preserves $S L(2, \mathbb{R})_{L} \times U(1)_{R}$ isometry, but this time the $U(1)_{R}$ corresponds to scaling transformations instead of translations. We will assume that the $w^{+}$-dependent deformation above is exactly marginal with respect to the left-moving conformal symmetry. This assumption could be verified using conformal perturbation theory, as in [39].

The gravity dual of the deformation on the right-hand side of (3.2) is naturally constructed in terms of the $w^{+}$-dependent $S L(2, \mathbb{R})_{L} \times U(1)_{R}$-invariant one-form on $A d S_{3}$, $\sigma_{0}$

$$
\sigma_{0}=\frac{w^{+} d w^{-}}{z^{2}}+\frac{d z}{z}
$$

where the $U(1)_{R}$ represents right-moving scaling transformations. Because $\sigma_{0}$ is not null, the background dual to the deformation will no longer be exact to second order in perturbation theory, as was true of the Schrödinger spacetimes [40]. Nevertheless, we can construct the solution order by order in perturbation theory ${ }^{6}$ in $\lambda T_{+}$

$$
d s_{\sigma_{0}}^{2}=\frac{1}{1-4 \lambda^{2} T_{+}^{2}}\left(d s_{A d S_{3}}^{2}-A_{\sigma_{0}} \otimes A_{\sigma_{0}}\right), \quad A_{\sigma_{0}}=2 \lambda T_{+} \ell \sigma_{0}
$$

The relationship between the parameter $\tilde{\lambda}$ introduced previously in (2.14) and the "true" coupling constant $\lambda$ of the dipole CFT is

$$
\tilde{\lambda}=\frac{2 \lambda}{\sqrt{1-4 \lambda^{2} T_{+}^{2}}}
$$

Performing the coordinate transformation

$$
w^{ \pm}=\sqrt{\frac{r-T_{+} T_{-}}{r+T_{+} T_{-}}} e^{2 T_{ \pm} x^{ \pm}}, \quad z=\sqrt{\frac{2 T_{+} T_{-}}{r+T_{+} T_{-}}} e^{T_{+} x^{+}+T_{-} x^{-}}
$$

we get precisely the warped black string background (2.14). This is the same coordinate transformation that takes Poincaré $\mathrm{AdS}_{3}$ into BTZ [47]. Note that, asymptotically, $w_{ \pm} \sim$ $e^{2 T_{ \pm} x^{ \pm}}$, as expected.

To summarize, our assumption (3.1) implies that from the point of view of the theory on the $x^{ \pm}$plane, the only operator source turned on is that of the defining operator. Each of the $x^{ \pm}$observers sees a thermal bath of particles and a non-zero expectation value of the stress tensor. Meanwhile, the Minkowski $w^{ \pm}$observer sees a non-trivial source for a $w^{+}$-dependent operator, whose coefficient depends on $T_{+}$. Thus, different thermal states in the dipole CFT, from the point of view of the Minkowski observer, correspond to different $S L(2, \mathbb{R})_{L}$-invariant irrelevant deformations of a $\mathrm{CFT}_{2}$ or, equivalently, to different exactly

\footnotetext{
${ }^{6}$ The identification of the coefficient of the boundary operator with the asymptotic value of the spacetime field, to all orders in perturbation theory, is based on the dictionary proposed in $[33,42,43]$. Note that the resulting gravity solution makes sense only if $2 \lambda T_{+}<1$. We are not sure whether this is a physical restriction on the theory - namely, that the inverse temperature cannot be smaller than the non-locality scale $\lambda$ - or the assumption that $\lambda$ is $T_{+}$-independent is wrong, or the all-orders holographic dictionary that we used is incorrect. In any case, as long as $\tilde{\lambda}=\lambda f\left(\lambda T_{+}\right)$with $f(0)=2$, the precise form of this holographic map does not affect the computations of section 1 .
} 
marginal deformations of a dipole CFT. Finally, from the bulk point of view, each $w^{+}$ dependent irrelevant deformation corresponds to a different non-normalizable X-mode, with different asymptotics. Any given black string background can be obtained by turning on a $T_{+}$-dependent non-normalizable (but nevertheless tractable) deformation of $\mathrm{AdS}_{3}$, followed by the coordinate transformation (3.6), which corresponds to a non-linear T-mode.

\section{Holography}

In the following, we would like to compute the expectation value of the holographic stress tensor in the warped black string backgrounds using holographic renormalization. Since from the point of view of each of the $x^{ \pm}$observers, only the stress tensor is turned on, we can compute its expectation value by simply coupling the theory to a background metric and then varying the on-shell action with respect to it. According to Papadimitriou's prescription, studying exclusively the stress tensor sector corresponds to solely turning on T-modes around a given black string spacetime.

Because backgrounds with different $T_{+}$are not diffeomorphic to each other, we will not be able to change the state of the theory by just turning on T-modes. Thus, we will be forced to work at fixed $T_{+}$. Nevertheless, for every fixed $T_{+}$, we will still be able to couple the theory to an arbitrary metric (at least in the classical analysis) and compute the expectation value of the stress tensor. We find that the holographic dictionary - and in particular the designation of the holographic sources - depends on $T_{+}$, and thus on the state of the theory. This is a joint consequence of Papadimitriou's prescription - which identifies T-modes around any given background with the stress tensor sector - and the unusual feature of Schrödinger spacetimes that different states correspond to different asymptotic behaviors, as is clear from the temperature-dependence of the coefficient of the deforming operator on the right-hand side of (3.2).

\subsection{The finite-temperature FG expansion}

For reasons of computational simplicity, as in [24], we restrict our study to T-modes only. To construct general T-modes above the black string backgrounds, we first note that the original black string metric (2.14) can be written as $^{7}$

$$
g_{\mu \nu}=\left(1+\mu^{2}\right)\left(\hat{g}_{\mu \nu}-A_{\mu} A_{\nu}\right), \quad \mu \equiv \tilde{\lambda} T_{+}
$$

where $\hat{g}_{\mu \nu}$ is the BTZ metric, satisfying

$$
\hat{R}_{\mu \nu}+\frac{2}{\ell^{2}} \hat{g}_{\mu \nu}=0
$$

and the background vector field $A_{\mu}$ solves

$$
F_{\mu \nu}=\frac{2}{\ell} \hat{\epsilon}_{\mu \nu \lambda} A^{\lambda}, \quad \hat{A}^{2}=\frac{\mu^{2}}{1+\mu^{2}}
$$

\footnotetext{
${ }^{7}$ The coefficient of the $A_{\mu} A_{\nu}$ term is fixed by the requirement that certain divergent terms cancel between $g_{\mu \nu}$ and $A_{\mu} A_{\nu}$. The coefficient of $\hat{g}_{\mu \nu}$ is fixed by demanding that the symplectic form not have any temperature-dependent overall factors.
} 
Since both the definition (4.1) and the equations of motion for $\hat{g}, A$ are covariant, it follows that the above equations hold for any background that is diffeomorphic to the original one. Thus, to find the most general T-mode solution around a given black string background one simply needs to solve (4.2) for $\hat{g}$, then solve (4.3) for $A$ and plug into (4.1). The constraint $\hat{A}^{2}=$ const. guarantees (at least at linearized order) that only pure gauge modes are solutions.

Writing the metric $\hat{g}$ in radial gauge

$$
d \hat{s}^{2}=d \eta^{2}+\hat{\gamma}_{i j} d x^{i} d x^{j}
$$

the general solution for $\hat{\gamma}$ is given by the Fefferman-Graham expansion [48]

$$
\hat{\gamma}_{i j}=e^{2 \eta / \ell} \hat{g}_{i j}^{(0)}+\hat{g}_{i j}^{(2)}+\frac{1}{4} e^{-2 \eta / \ell} \hat{g}_{i}^{(2) k} \hat{g}_{k j}^{(2)}
$$

where all indices are raised and lowered using the boundary metric $\hat{g}^{(0)}$. The asymptotic equations of motion imply that

$$
\hat{\nabla}_{i} \hat{g}_{j}^{(2) i}=\hat{\nabla}_{j} \hat{g}_{k}^{(2) k}, \quad \hat{g}_{k}^{(2) k}=-\frac{1}{2} \hat{R}\left[\hat{g}^{(0)}\right]
$$

Note that if $\hat{g}_{i j}^{(2)}$ has a generic dependence on $x^{i}$, then it is entirely determined by $\hat{g}_{i j}^{(0)}$, albeit in a very non-local fashion. If the solution considered is constant in $x^{i}$, then the trace of $\hat{g}^{(2)}$ needs to vanish, but the remaining components are independent of $\hat{g}^{(0)}$.

One may notice that (4.3) represent four equations for the field $A_{\mu}$, which only has three components. While the system seems overdetermined, a solution is always guaranteed to exist, because all solutions can be induced by a diffeomorphism. Nevertheless, one cannot solve for $A_{\mu}$ for an arbitrary background metric $\hat{g}_{\mu \nu}$, one needs to put the latter at least partially on-shell. More precisely, one needs to use multiples of (4.6) in order to satisfy all equations $A$ obeys, but never needs to solve for the the non-local relationship between $\hat{g}^{(0)}$ and $\hat{g}^{(2)}$. In particular, for the case of constant sources, the full solution for both $\hat{g}$ and $A$ is parametrized by five independent constants. For non-constant sources, the leading term in the $A_{\mu}$ expansion bears a rather non-local relation to the boundary data $\hat{g}^{(0)}$.

Given the radial $\mathrm{AdS}_{3}$ metric (4.4), one finds that the corresponding warped $\mathrm{AdS}_{3}$ metric reads ${ }^{8}$

$$
d s^{2}=N^{2} d \eta^{2}+\gamma_{i j}\left(d x^{i}+N^{i} d \eta\right)\left(d x^{j}+N^{j} d \eta\right)
$$

with

$$
\gamma_{i j}=\left(1+\mu^{2}\right)\left(\hat{\gamma}_{i j}-A_{i} A_{j}\right), \quad N_{i}=-\left(1+\mu^{2}\right) A_{i} A_{\eta}, \quad N^{2}=\frac{1+\mu^{2}}{1+\left(1+\mu^{2}\right) A_{\eta}^{2}}
$$

${ }^{8}$ For future reference, we also have

$$
\gamma^{i j}=\frac{1}{1+\mu^{2}} \hat{\gamma}^{i j}+\frac{\hat{A}^{i} \hat{A}^{j}}{1+\left(1+\mu^{2}\right) A_{\eta}^{2}}, \quad \operatorname{det} \gamma=\left(1+\mu^{2}\right)\left(1+\left(1+\mu^{2}\right) A_{\eta}^{2}\right) \operatorname{det} \hat{\gamma}
$$


Unless $A_{\eta}=0$, if the $\mathrm{AdS}_{3}$ metric is in radial gauge, then the warped $\mathrm{AdS}_{3}$ one will not be, and vice-versa. Since in holographic renormalization it is natural to have the boundary at fixed radial coordinate, we need to bring the warped $\mathrm{AdS}_{3}$ metric to radial gauge via a coordinate transformation. At linearized level above the black hole background, it reads

$$
x^{i} \rightarrow x^{i}+\xi^{i}\left(\eta, x^{i}\right), \quad \xi^{i}=\left(1+\mu^{2}\right) \int d \eta A^{i} A_{\eta}
$$

In the explicit linearized solution, $A_{\eta}$ is $\eta$-independent, as is the zeroth order $A^{i}$. Consequently, one can integrate (4.10) to find

$$
\xi^{i}=\eta N^{2} A^{i} A_{\eta}
$$

One can then show that the radial symplectic form, when evaluated the general linearized T-mode solution, equals the radial symplectic form of the auxiliary $\operatorname{AdS}_{3}$ spacetime ${ }^{9}$. By Papadimitriou's prescription, we choose the holographic sources to be $\hat{g}_{i j}^{(0)}$.

\subsection{Holographic renormalization}

To perform holographic renormalization, we need to compute the on-shell action for the general T-modes, regulate it and then remove the divergences. The on-shell action consists of a bulk piece, which is given by (2.13) evaluated on the general T-mode solution

$$
S_{\text {bulk }}=-\frac{1}{16 \pi G_{3}} \int d^{3} x \frac{4}{\ell^{2}\left(1+\mu^{2}\right)} \sqrt{g}=-\frac{1}{16 \pi G_{3}} \int d^{3} x \frac{4}{\ell^{2}} \sqrt{\hat{g}}
$$

and a Gibbons-Hawking boundary term ${ }^{10}$

$$
S_{G H}=\frac{1}{8 \pi G_{3}} \int d^{2} x K \sqrt{\gamma}
$$

Let us ignore for a moment the final diffeomorphism needed to bring the warped $\mathrm{AdS}_{3}$ metric into radial gauge. In this case, (4.12) shows that the bulk on-shell action is identical to that of the auxiliary $\mathrm{AdS}_{3}$ spacetime. One can also easily compute the extrinsic curvature $K$ from its definition

$$
K_{i j}=-\frac{1}{2 N}\left(\partial_{\eta} \gamma_{i j}-D_{i} N_{j}-D_{j} N_{i}\right)
$$

where $D_{i}$ is the covariant derivative associated to the metric $\gamma_{i j}$. Plugging in the expressions for $N, N_{i}$, we find

$$
K_{i j}=\frac{\left(1+\mu^{2}\right)}{N} \hat{K}_{i j}-\frac{N}{2}\left(1+\mu^{2}\right)\left(\hat{D}_{i} A_{j}+\hat{D}_{j} A_{i}\right) A_{\eta}+\frac{N}{\ell}\left(A_{i} \hat{\epsilon}_{j}^{k}+A_{j} \hat{\epsilon}_{i}^{k}\right) A_{k}
$$

\footnotetext{
${ }^{9}$ To show this, one needs to use various multiples of the linearized asymptotic equations of motion (4.6). The calculation is almost identical to that in section 2 of [24].

${ }^{10}$ Since ultimately we would like to keep the metric $\hat{g}$ fixed at the boundary rather than $g$, it is not clear whether the Gibbons-Hawking boundary term above is the one required by the modified variational principle. We decided to add it, nevertheless, because we would like to view holographic renormalization as a canonical transformation between the original variables $\left(\gamma_{i j}, \pi^{i j}\right)$ and the new ones $\left(\hat{\gamma}_{i j}, \hat{\pi}^{i j}\right)$, and we require the starting action to have a well-defined variational principle.
} 
Taking the trace, one can show that

$$
K \sqrt{\gamma}=\hat{K} \sqrt{\hat{\gamma}}-N^{2} \hat{A}^{i} \partial_{i} A_{\eta} \sqrt{\hat{\gamma}}
$$

at full nonlinear level. Thus, barring the issues involving the final diffeomorphism, the on-shell action for T-modes in a warped black string background is identical to that of the auxiliary $\mathrm{AdS}_{3}$ spacetime used to construct the T-modes, up to the last term in (4.16). To render the action finite, we simply need to subtract this term and include an additional counterterm proportional to $\int \sqrt{\hat{\gamma}}$, as in $\mathrm{AdS}_{3}$. Because the equations of motion for $A$ - which we used extensively in manipulating the above expressions - do not impose any non-local constraint between the asymptotic metric coefficients, the resulting renormalized on-shell action will be the same functional of $\hat{g}^{(0)}, \hat{g}^{(2)}$ as the $\mathrm{AdS}_{3}$ one [48], and therefore it will have the same variation.

To include the effect of the diffeomorphism that reinstates the radial gauge, we need to work at infinitesimal level above a background that has $A_{\eta}=0$. It is not hard to show that the first order contribution of $\xi^{i}$ to both the bulk and the boundary action vanishes. Higher order contributions in $\xi^{i}$ will generically be non-zero; nevertheless, since $\xi^{i}$ is proportional to $\eta=-\ell \log z$, the logarithm of the usual radial coordinate, the corrections to the on-shell action due to these terms will be purely logarithmic in perturbation theory and can be renormalized separately from the power law divergences.

It would be interesting to understand how the canonical momenta transform under the change of generalized coordinates from $(\gamma, A)$ to $\hat{\gamma}$. For this, we need to study the variation of the on-shell action. Since the computation becomes rather cumbersome in the general case, in the following we will only study two simpler ones: the constant non-linear case and that of a general linearized perturbation around a given black string background.

\section{$\diamond$ The constant case}

In the constant (i.e. $x^{i}$-independent) case, both $g$ and $\hat{g}$ are in radial gauge, since

$$
A_{\eta}=-\frac{\ell}{4} \hat{\epsilon}^{i j} F_{i j}=0 \quad \Rightarrow \quad N_{i}=0, \quad N=\sqrt{1+\mu^{2}}
$$

Therefore, we do not need to worry about the position of the radial cutoff surface. Setting $16 \pi G_{3}=1$ for now, the variation of the on-shell action reads

$$
\begin{aligned}
\delta S_{\text {on-shell }} & =-\int d^{2} x \sqrt{\gamma}\left(\left(K^{i j}-K \gamma^{i j}\right) \delta \gamma_{i j}+\frac{2}{\ell} \epsilon^{i j} A_{i} \delta A_{j}\right)= \\
& =-\int d^{2} x \sqrt{\hat{\gamma}}\left[N^{3}\left(K^{i j}-K \gamma^{i j}\right) \delta \hat{\gamma}_{i j}+2\left(\frac{1}{\ell} \hat{\epsilon}^{i j}-N^{3}\left(K^{i j}-K \gamma^{i j}\right)\right) A_{i} \delta A_{j}\right]
\end{aligned}
$$

The terms proportional to $\delta A_{i}$ in the above variation are non-local in the boundary data

$\hat{\gamma}$, and they need to cancel from the final answer, for consistency. Using the simplified equations of motion for $A_{i}$

$$
\partial_{\eta} A_{i}=\frac{2}{\ell} \hat{\epsilon}_{i j} \hat{A}^{j}, \quad A_{i} \hat{A}^{i}=\frac{\mu^{2}}{1+\mu^{2}}
$$


one can show that the extrinsic curvature satisfies the following relations ${ }^{11}$

$$
\hat{K}_{i j} \hat{A}^{j}=-\frac{1}{\ell} \hat{\epsilon}_{i j} \hat{A}^{j}, \quad K^{i j}=N^{-3} \hat{K}^{i j}, \quad K=N^{-1} \hat{K}
$$

Using the above equations, we can show that

$$
\begin{aligned}
\delta S & =-\int d^{2} x \sqrt{\hat{\gamma}}\left[\left(\hat{K}^{i j}-\hat{K} \hat{\gamma}^{i j}-N^{2} \hat{K} \hat{A}^{i} \hat{A}^{j}\right) \delta \hat{\gamma}_{i j}+2 N^{2} \hat{K} \hat{A}^{j} \delta A_{j}\right] \\
& =-\int d^{2} x \sqrt{\hat{\gamma}}\left[\left(\hat{K}^{i j}-\hat{K} \hat{\gamma}^{i j}\right) \delta \hat{\gamma}_{i j}+N^{2} \hat{K} \delta\left(\hat{A}^{i} A_{i}\right)\right]
\end{aligned}
$$

The last term vanishes by virtue of the fact that $\hat{A}^{2}$ is a constant. Thus, the T-mode part of the variation of the action is only proportional to $\delta \hat{\gamma}_{i j}$, and the terms non-local in $\delta \hat{\gamma}_{i j}$ have canceled away completely. In showing this, we only needed to use the equations of motion for the quantities multiplying the variations and did not add any counterterms. From the point of view of the canonical variables, we simply made the replacement

$$
\gamma_{i j}, A_{i} \longrightarrow \hat{\gamma}_{i j}=N^{-2} \gamma_{i j}+A_{i} A_{j}, A_{i}
$$

As a consequence, the canonical momenta changed to

$$
\pi^{i j} \rightarrow \pi^{i j}=N^{-2} \hat{\pi}^{i j}-\sqrt{\hat{\gamma}} \hat{K} \frac{\delta \mathcal{C}}{\delta \hat{\gamma}_{i j}}, \quad \pi_{A}^{i} \rightarrow \pi_{A}^{i}-2 N^{2} \pi^{i j} A_{j}=-N^{2} \sqrt{\hat{\gamma}} \hat{K} \frac{\delta \mathcal{C}}{\delta A_{i}}
$$

where $\mathcal{C}=\hat{A}^{i} A_{i}-\mu^{2} /\left(1+\mu^{2}\right)=0$ is the constraint that the canonical variables satisfy and the canonical momenta can be read off from (4.18). Adding the vanishing quantity $N^{2} \hat{K} \mathcal{C} \sqrt{\hat{\gamma}}$ to the action, we find that the momentum conjugate to $\hat{\gamma}_{i j}$ becomes $\hat{\pi}^{i j}$, where

$$
\hat{\pi}^{i j}=-\sqrt{\hat{\gamma}}\left(\hat{K}^{i j}-\hat{K} \hat{\gamma}^{i j}\right)
$$

while the canonical momentum multiplying $\delta A_{i}$ vanishes. It would be interesting to check whether the above transformations correspond indeed to a canonical transformation in the constrained theory. To obtain the final renormalized momentum, one needs to add, as in $\mathrm{AdS}_{3}$, a boundary cosmological constant counterterm, which renders the on-shell action finite. It also shifts $\hat{\pi}^{i j} \rightarrow \hat{\pi}^{i j}-\ell^{-1} \hat{\gamma}^{i j} \sqrt{\hat{\gamma}}$, thus making the map between the phase space variables $\hat{\gamma}_{i j}, \hat{\pi}^{i j}$ and the asymptotic constants $\hat{g}_{i j}^{(0)}, \hat{g}^{(2) i j}$ asymptotically diagonal [23].

\section{$\diamond$ The non-constant case}

We now consider linearized perturbations of the black string background (2.14). Since $A_{\eta}$ no longer vanishes, we need to take into account the effect of the diffeomorphism $\xi^{i}$ given by (4.11). After applying the diffeomorphism, the various quantities that enter the on-shell action, now denoted with a tilde, read

$$
\tilde{\pi}^{i j}=\pi^{i j}-\partial_{k} \xi^{i} \pi^{k j}-\partial_{k} \xi^{j} \pi^{i k}+\xi^{k} \partial_{k} \pi^{i j}, \quad \tilde{\gamma}_{i j}=\gamma_{i j}+D_{i} \xi_{j}+D_{j} \xi_{i}
$$

\footnotetext{
${ }^{11}$ The first relation follows the fact that both $\hat{K}_{i j} \hat{A}^{j}$ and $\hat{\epsilon}_{i j} \hat{A}^{j}$ are normal to $A_{i}$ and their norm is constant. The norm can be computed using the equation of motion for $\hat{K}_{i j}: \partial_{\eta} \hat{K}^{i j}=\hat{R}^{i j}{ }^{(2)} \hat{R}^{i j}+\hat{K} \hat{K}^{i j}+2 \hat{K}^{i k} \hat{K}_{k}{ }^{j}$.
} 


$$
\tilde{A}_{i}=A_{i}+\partial_{i}\left(\xi^{k} A_{k}\right)+\xi^{k} F_{k i}, \quad \tilde{A}_{\eta}=A_{\eta}
$$

Note that the last term in $\tilde{\pi}^{i j}$ and $\tilde{A}^{i}$ is second order in derivatives and can be dropped. The variation of the on-shell action is

$$
\delta S=-\int d^{2} x \sqrt{\gamma}\left(\left(\tilde{K}^{i j}-\tilde{K} \tilde{\gamma}^{i j}\right) \delta \tilde{\gamma}_{i j}+\frac{2}{\ell} \tilde{\epsilon}^{i j} \tilde{A}_{i} \delta \tilde{A}_{j}\right)=\delta_{0} S+\delta_{1} S+\delta_{2} S
$$

where $\delta_{0} S$ represents the action variation before including the diffeomorphism, $\delta_{1} S$ is linear in $\xi^{i}$, while $\delta_{2} S$ is quadratic. Of them, only

$$
\delta_{0} S=-\int d^{2} x \sqrt{\gamma}\left(\left(K^{i j}-K \gamma^{i j}\right) \delta \gamma_{i j}+\frac{2}{\ell} \epsilon^{i j} A_{i} \delta A_{j}\right)
$$

contributes to the finite piece of the action. The remaining contributions only have logarithmic divergences and can be renormalized separately ${ }^{12}$. Noting that, at linearized level

$$
\hat{K}_{i j} \hat{A}^{j}+\partial_{i} A_{\eta}=-\frac{1}{\ell} \hat{\epsilon}_{i j} \hat{A}^{j}
$$

we can show that the expression for the on-shell action can be simplified to

$$
\begin{gathered}
\delta_{0} S=-\int d^{2} x \sqrt{\hat{\gamma}}\left[\left(\hat{K}^{i j}-\hat{K} \hat{\gamma}^{i j}\right) \delta \hat{\gamma}_{i j}+N^{2} \hat{K} \delta\left(\hat{A}^{i} A_{i}\right)+2 N^{2} \hat{D}_{k} \hat{A}^{k} \delta A_{\eta}\right]- \\
-2 N^{2} \int d^{2} x \delta\left(\hat{A}^{k} \partial_{k} A_{\eta} \sqrt{\hat{\gamma}}\right)
\end{gathered}
$$

Since $A_{\mu}$ is divergence-free, we have

$$
\hat{D}_{k} \hat{A}^{k}=\hat{K} A_{\eta}-\partial_{\eta} A_{\eta}
$$

Plugging in, we find

$$
\begin{gathered}
\delta_{0} S=-\int d^{2} x \sqrt{\hat{\gamma}}\left[\left(\hat{K}^{i j}-\hat{K} \hat{\gamma}^{i j}\right) \delta \hat{\gamma}_{i j}+N^{2} \hat{K} \delta\left(\hat{A}^{2}\right)-2 N^{2} \partial_{\eta} A_{\eta} \delta A_{\eta}\right]- \\
-2 N^{2} \int d^{2} x \delta\left(\hat{A}^{k} \partial_{k} A_{\eta} \sqrt{\hat{\gamma}}\right)
\end{gathered}
$$

The first term is identical to the variation of the on-shell action in $\mathrm{AdS}_{3}$, the second is zero upon imposing the constraint, the third vanishes identically in perturbation theory because $\partial_{\eta} A_{\eta}=0$, and the forth agrees precisely with the linearization of (4.16). To render the action finite, we need to add the counterterms

$$
S_{c t}=2 N^{2} \int d^{2} x \hat{A}^{k} \partial_{k} A_{\eta} \sqrt{\hat{\gamma}}-\frac{2}{\ell} \int d^{2} x \sqrt{\hat{\gamma}}
$$

It would be interesting to understand whether the addition of the above counterterms induces a canonical transformation in the constrained theory that describes the T-modes.

\footnotetext{
${ }^{12}$ Using extensively the fact that we have a linearized solution around an $x^{i}$-independent background, it is possible to show that $\delta_{2} S=2 \eta^{2} \mu^{2} N^{2} \int d^{2} x \sqrt{\gamma} \pi^{i k} \partial_{i} A_{\eta} \delta \partial_{k} A_{\eta}$ and thus can be canceled by a counterterm proportional to $(\log z)^{2}$ that is linear in the extrinsic curvature. We could not easily bring $\delta_{1} S$ to any nice form, but it is very possible that counterterms linear the holographic momenta will also be necessary.
} 


\subsection{The holographic stress tensor}

Since the renormalized on-shell action in the warped black string backgrounds equals the one in the auxiliary $\mathrm{AdS}_{3}$ and the boundary data are the same, it follows that the holographic stress tensor is the same as the $\mathrm{AdS}_{3}$ one

$$
\left\langle T_{i j}\right\rangle=-\frac{2}{\sqrt{\hat{g}^{(0)}}} \frac{\delta S_{r e n}\left[\hat{g}^{(0)}\right]}{\delta \hat{g}^{(0) i j}}=\frac{\ell}{8 \pi G_{3}}\left(\hat{g}_{i j}^{(2)}-\hat{g}_{i j}^{(0)} \hat{g}_{k}^{(2) k}\right)
$$

Using the asymptotic relations (4.6) between $\hat{g}^{(2)}$ and $\hat{g}^{(0)}$, we find the holographic Ward identities

$$
\hat{\nabla}_{i} \hat{T}^{i j}=0, \quad \hat{T}_{i}^{i}=\frac{\ell}{16 \pi G_{3}} \hat{R}\left[\hat{g}^{(0)}\right]
$$

The fact that the stress tensor is both symmetric and conserved is indicative of an underlying relativistic structure of this sector of the theory, which is consistent with the existence of both left-moving and right-moving Virasoro extensions of the asymptotic symmetry group [31,32]. The conformal anomaly read off from the above equation

$$
c=\frac{3 \ell}{2 G_{3}}
$$

agrees with the result (2.17) obtained using asymptotic symmetry group analyses. We can also compute the expectation value of the holographic stress tensor on the black string backgrounds

$$
\left\langle T_{++}\right\rangle=\frac{\ell}{8 \pi G_{3}} T_{+}^{2}, \quad\left\langle T_{--}\right\rangle=\frac{\ell}{8 \pi G_{3}} T_{-}^{2}
$$

The energy and momentum of the string are given by

$$
Q_{\xi}=\int d x \hat{n}^{i}\left\langle T_{i j}\right\rangle \xi^{j} \sqrt{\hat{\sigma}}, \quad \xi^{i}=\partial_{\tau}, \partial_{x}
$$

where the coordinates $x, \tau$ are defined via

$$
x^{ \pm}=x \pm \tau
$$

$\hat{n}^{i}$ is the unit normal to the surface of constant $\tau$ at the boundary (using the metric $\hat{\gamma}$ ) and $\hat{\sigma}$ is the induced metric on this surface. It is not hard to show that $Q_{\tau}$ and $Q_{x}$ per unit $x$-length equal precisely the energy and momentum per unit length of the string (2.16), which were computed in [32] using covariant methods.

It is quite remarkable that a simple, linear expression such as (4.38) can capture the conserved charges, which in the covariant formalism receive non-linear contributions that are strongly background-dependent. Note that the conserved charges (4.38) effectively "live" in the auxiliary $\mathrm{AdS}_{3}$ spacetime used for the construction of the T-modes, whose definition depends on the value of $T_{+}$. It is plausible that by formulating the problem in terms of the auxiliary $\mathrm{AdS}_{3}$ spacetime, the non-linear charges in warped $\mathrm{AdS}_{3}$ that one always encounters in the covariant formalism are traded for a set of linear $\operatorname{charges}^{13}$, but with a state-dependent dictionary (4.1).

\footnotetext{
${ }^{13}$ We thank Tom Hartman for making this point.
} 


\subsection{General warped black strings}

It is not hard to extend these results to the general four-parameter family of warped black strings in type IIB supergravity with RR three-form flux found in [32]. These sixdimensional black string solutions depend as before on two parameters $T_{ \pm}$related to their Hawking temperature and horizon velocity via (2.15). In addition, they have two warping parameters $\lambda_{1,2}$, which correspond to two distinct massive vector fields in spacetime, and consequently two different $(1,2)$ operators that define the boundary theory.

The solutions have a somewhat complicated dependence on the two parameters $\lambda_{1,2}$, so we will not reproduce them here ${ }^{14}$. Nevertheless, the energy and momentum per unit length of any given black string take the following simple form [32]

$$
E \pm P=\frac{\pi \hat{\ell}^{4}}{2 G_{6}} T_{ \pm}^{2}
$$

where $\hat{\ell}^{4}$ equals the product of the electric and magnetic charges of the string. The asymptotic symmetry group computations of [32] yield a Virasoro algebra with central charge

$$
c=\frac{3 \pi^{2} \hat{\ell}^{4}}{G_{6}}
$$

We would like to reproduce these values from a holographic stress tensor computation.

The six-dimensional black string solutions of [32] cannot in general be captured by a three-dimensional consistent truncation of type IIB string theory; nevertheless, if we only consider T-modes about a given black string background with temperature $T_{+}$, then such a truncation is possible. Upon the dimensional reduction, the three-dimensional Einstein metric can be written as

$$
d s_{3}^{2}=\left(1+\mu^{2}\right) d s_{B T Z}^{2}-\mu^{2} \hat{\ell}^{2} \sigma_{0}^{2}, \quad \mu=\tilde{\lambda} T_{+}
$$

where the BTZ radius is $\hat{\ell}$ and the relationship between the six- and three-dimensional Newton constants is $G_{6}=2 \pi^{2} \hat{\ell}^{3} G_{3}$. The quantity $\tilde{\lambda}$ is defined in [32] and depends on $\lambda_{1}, \lambda_{2}$ and $T_{+}$in a rather complicated manner. The two massive vector fields that support the solution are both proportional to an auxiliary massive vector field satisfying (4.3) and given by

$$
A_{\mu}=\frac{\mu \hat{\ell}}{\sqrt{1+\mu^{2}}} \sigma_{0}
$$

The $S L(2, \mathbb{R})_{L} \times U(1)_{R}$-invariant one-form $\sigma_{0}$ needs to be written in black hole coordinates, using (3.6). The reason that $\hat{\ell}$, rather than any other length scale, is singled out is because the on-shell six-dimensional action is proportional to $\hat{\ell}^{4}$ and thus it is the natural length parameter to keep fixed in the reduction.

\footnotetext{
${ }^{14}$ They can be found in the equations $(2.19)-(2.24)$ in [32], whose notation we are using. The length $\hat{\ell}$ is defined in (3.23) of the same reference.
} 
We now define the T-modes as before in (4.1) - (4.3), with the only change that we replace $\ell$ by $\hat{\ell}$. Using the explicit solutions of [32], we can show that the on-shell bulk action, when evaluated on any T-mode solution, equals

$$
S_{b u l k}=-\frac{1}{16 \pi G_{3}} \int d^{3} x \sqrt{\hat{g}} \frac{4}{\hat{\ell}^{2}}
$$

The boundary action is the usual Gibbons-Hawking term and holographic renormalization on the truncated T-mode sector works identically as before. Thus, we obtain again a stress tensor of the form (4.34) that satisfies the holographic Ward identities (4.35), with conformal anomaly

$$
c=\frac{3 \hat{\ell}}{2 G_{3}}=\frac{3 \pi^{2} \hat{\ell}^{4}}{G_{6}}
$$

and holographic expectation values

$$
\left\langle T_{++}\right\rangle=\frac{\hat{\ell}}{8 \pi G_{3}} T_{+}^{2}=\frac{1}{2}(E+P), \quad\left\langle T_{--}\right\rangle=\frac{\hat{\ell}}{8 \pi G_{3}} T_{-}^{2}=\frac{1}{2}(E-P)
$$

again in perfect agreement with the covariant charge computations of [32].

\section{Discussion}

The goal of this article was to find the holographic interpretation of the general warped black strings described in [32], i.e. to spell out which operator sources and expectation values are turned on in these backgrounds. The holographic dictionary we have proposed is based upon Papadimitriou's prescription [23] and a certain reasonable assumption (3.1) about the behaviour of finite-temperature dipole CFTs. Our holographic computation of the conserved charges and anomalies agrees with previous results obtained using covariant methods [32].

The warped $\mathrm{AdS}_{3}$ holographic dictionary we have proposed has a number of curious features, such as the state-dependent identification of the holographic sources. It would be interesting to better understand this trait. Also, we need to better study the structure of the counterterms necessary for holographic renormalization. For example, in AdS the counterterms need to be constructed from the induced fields at the boundary and their derivatives [7]. For spacetimes corresponding to irrelevant deformations of AdS, [33,42,43] argued that the counterterms can also include the conjugate momenta (i.e. the extrinsic curvature), but only at quadratic order or higher. It would be interesting to understand the general rules for constructing counterterms in warped $\mathrm{AdS}_{3}$, renormalized according to the prescription of [23].

Another interesting exercise would be to check whether holographic renormalization in warped $\mathrm{AdS}_{3}$ - with holographic sources chosen à la Papadimitriou - corresponds indeed to a canonical transformation, thus following the pattern described in [23]. Should this be the case, it would be a non-trivial check that the prescription is self-consistent even in a case where its predictions are different from those obtained using other methods $[11,33]$. For better understanding this issue, it may be useful to also include the X-modes in the analysis, which can be easily done, at least at linearized level. 
Although we have been able to find agreement between the energy and momentum density of the black strings computed using the holographic versus the covariant method, the relationship between the two formalisms is far from clear. For example, the holographic method uses the auxiliary $\mathrm{AdS}_{3}$ metric $\hat{\gamma}$ instead of the induced metric, $\gamma$, at the boundary, thus completely obscuring the conservation of the charges from the point of view of warped $\mathrm{AdS}_{3}$. On the other hand, the holographic method yields charges that are linear in the stress tensor and take a very simple form in the auxiliary $\mathrm{AdS}_{3}$ spacetime, to be compared with the very non-linear expressions for the charges in the covariant formalism. It would thus be very interesting to understand the precise relationship between the two formalisms and use it to understand e.g. the Kerr/CFT correspondence - where the covariant methods of [41] have been particularly successful - from a holographic point of view.

Finally, one should ascertain whether Papadimitriou's prescription, which has led to the above rather interesting and intriguing results, is also the correct one. This question can only be answered by understanding the relationship between the spacetime fields and the field theory gauge-invariant operators. The latter may be possible to construct using the prescription of [49] for non-commutative field theories, and the couplings to the spacetime metric may be found using the methods of $[37,38]$. Should the answer be affirmative, it would have implications not only for warped $\mathrm{AdS}_{3}$ holography and the Kerr/CFT correspondence, but it would also give us confidence that Papadimitriou's prescription is the correct general one.

\section{Acknowledgements}

The author is grateful to Tom Hartman, Juan Maldacena, Ioannis Papadimitriou, Andrew Strominger and Marika Taylor for interesting conversations, and would like to especially thank Tom Hartman for useful comments on the draft. This work was supported in part by the DOE grant DE-SC0007901.

\section{References}

[1] G. 't Hooft, "Dimensional reduction in quantum gravity", [gr-qc/9310026].

[2] J. M. Maldacena, "The Large $N$ limit of superconformal field theories and supergravity", Adv. Theor. Math. Phys. 2, 231 (1998), [hep-th/9711200].

[3] O. Aharony, S. S. Gubser, J. M. Maldacena, H. Ooguri and Y. Oz, "Large N field theories, string theory and gravity", Phys. Rept. 323, 183 (2000), [hep-th/9905111].

[4] E. Witten, "Anti-de Sitter space and holography", Adv. Theor. Math. Phys. 2, 253 (1998) [hep-th/9802150].

[5] S. S. Gubser, I. R. Klebanov and A. M. Polyakov, "Gauge theory correlators from noncritical string theory", Phys. Lett. B 428, 105 (1998) [hep-th/9802109].

[6] S. de Haro, S. N. Solodukhin and K. Skenderis, "Holographic reconstruction of space-time and renormalization in the AdS/CFT correspondence", Commun. Math. Phys. 217, 595 (2001), [hep-th/0002230].

[7] K. Skenderis, "Lecture notes on holographic renormalization", Class. Quant. Grav. 19, 5849 (2002), [hep-th/0209067]. 
[8] S. F. Ross, "Holography for asymptotically locally Lifshitz spacetimes," Class. Quant. Grav. 28, 215019 (2011), arXiv: 1107.4451 [hep-th].

[9] R. B. Mann and R. McNees, "Holographic Renormalization for Asymptotically Lifshitz Spacetimes," JHEP 1110, 129 (2011), arXiv: 1107.5792 [hep-th].

[10] M. Baggio, J. de Boer and K. Holsheimer, "Hamilton-Jacobi Renormalization for Lifshitz Spacetime," JHEP 1201, 058 (2012), arXiv: 1107.5562 [hep-th].

[11] S. F. Ross and O. Saremi, "Holographic stress tensor for non-relativistic theories," JHEP 0909, 009 (2009), arXiv: 0907.1846 [hep-th].

[12] Y. Korovin, K. Skenderis and M. Taylor, "Lifshitz as a deformation of Anti-de Sitter," arXiv: 1304.7776 [hep-th].

[13] T. Wiseman and B. Withers, "Holographic renormalization for coincident Dp-branes," JHEP 0810, 037 (2008), arXiv: 0807.0755 [hep-th].

[14] I. Kanitscheider, K. Skenderis and M. Taylor, "Precision holography for non-conformal branes," JHEP 0809, 094 (2008), arXiv: 0807.3324 [hep-th].

[15] O. Aharony, "The NonAdS/nonCFT correspondence, or three different paths to QCD," [hep-th/0212193].

[16] J. Erdmenger, N. Evans, I. Kirsch and E. Threlfall, "Mesons in Gauge/Gravity Duals - A Review,” Eur. Phys. J. A 35, 81 (2008), arXiv: 0711.4467 [hep-th].

[17] A. Zaffaroni, "RTN lectures on the non AdS / non CFT correspondence," PoS RTN 2005, 005 (2005).

[18] For further references, please consult the above reviews.

[19] D. T. Son, "Toward an AdS/cold atoms correspondence: A Geometric realization of the Schrödinger symmetry," Phys. Rev. D 78 (2008) 046003, arXiv: 0804.3972 [hep-th].

[20] K. Balasubramanian and J. McGreevy, "Gravity duals for non-relativistic CFTs," Phys. Rev. Lett. 101 (2008) 061601, arXiv: 0804.4053 [hep-th].

[21] M. Guica, T. Hartman, W. Song and A. Strominger, "The Kerr/CFT Correspondence," Phys. Rev. D 80, 124008 (2009), arXiv: 0809.4266 [hep-th].

[22] S. El-Showk and M. Guica, "Kerr/CFT, dipole theories and nonrelativistic CFTs," JHEP 1212 (2012) 009, arXiv: 1108.6091 [hep-th].

[23] I. Papadimitriou, "Holographic renormalization as a canonical transformation," JHEP 1011, 014 (2010), arXiv: 1007.4592 [hep-th].

[24] M. Guica, “A Fefferman-Graham-Like Expansion for Null Warped AdS(3)," JHEP 1212 084, (2012), arXiv: 1111.6978 [hep-th].

[25] M. Alishahiha and O. J. Ganor, "Twisted backgrounds, PP waves and nonlocal field theories," JHEP 0303, 006 (2003), [hep-th/0301080].

[26] J. Maldacena, D. Martelli and Y. Tachikawa, "Comments on string theory backgrounds with non-relativistic conformal symmetry," JHEP 0810 (2008) 072, arXiv: 0807.1100 [hep-th].

[27] C. P. Herzog, M. Rangamani and S. F. Ross, "Heating up Galilean holography," JHEP 0811, 080 (2008), arXiv: 0807.1099 [hep-th]. 
[28] A. Adams, K. Balasubramanian and J. McGreevy, "Hot Spacetimes for Cold Atoms," JHEP 0811, 059 (2008), arXiv: 0807.1111 [hep-th].

[29] D. Anninos, W. Li, M. Padi, W. Song and A. Strominger, "Warped AdS $S_{3}$ Black Holes," JHEP 0903, 130 (2009), arXiv: 0807.3040 [hep-th].

[30] G. Compere and S. Detournay, "Semi-classical central charge in topologically massive gravity," Class. Quant. Grav. 26, 012001 (2009), [Erratum-ibid. 26, 139801 (2009)], arXiv: 0808.1911 [hep-th].

[31] W. Song and A. Strominger, "Warped AdS 3 /Dipole-CFT Duality," JHEP 1205, 120 (2012), arXiv: 1109.0544 [hep-th].

[32] S. Detournay and M. Guica, "Stringy Schroedinger truncations," arXiv: 1212.6792 [hep-th].

[33] B. C. van Rees, "Correlation functions for Schrödinger backgrounds," arXiv: 1206.6507 [hep-th].

[34] A. Bergman and O. J. Ganor, "Dipoles, twists and noncommutative gauge theory," JHEP 0010, 018 (2000), [hep-th/0008030].

[35] A. Bergman, K. Dasgupta, O. J. Ganor, J. L. Karczmarek and G. Rajesh, "Nonlocal field theories and their gravity duals," Phys. Rev. D 65, 066005 (2002), [hep-th/0103090].

[36] K. Dasgupta and M. M. Sheikh-Jabbari, "Noncommutative dipole field theories," JHEP 0202, 002 (2002), [hep-th/0112064].

[37] Y. Okawa and H. Ooguri, "How noncommutative gauge theories couple to gravity," Nucl. Phys. B 599, 55 (2001), [hep-th/0012218].

[38] H. Liu and J. Michelson, "Supergravity couplings of noncommutative D-branes," Nucl. Phys. B 615, 169 (2001), [hep-th/0101016].

[39] M. Guica, K. Skenderis, M. Taylor and B. C. van Rees, "Holography for Schrödinger backgrounds," JHEP 1102 (2011) 056, arXiv: 1008.1991 [hep-th].

[40] P. Kraus and E. Perlmutter, "Universality and exactness of Schrödinger geometries in string and M-theory," JHEP 1105 (2011) 045, arXiv: 1102.1727 [hep-th].

[41] G. Barnich and F. Brandt, "Covariant theory of asymptotic symmetries, conservation laws and central charges," Nucl. Phys. B 633, 3 (2002), [hep-th/0111246].

[42] B. C. van Rees, "Holographic renormalization for irrelevant operators and multi-trace counterterms," JHEP 1108 (2011) 093, arXiv: 1102.2239 [hep-th].

[43] B. C. van Rees, "Irrelevant deformations and the holographic Callan-Symanzik equation," JHEP 1110, 067 (2011), arXiv: 1105.5396 [hep-th].

[44] V. Balasubramanian and P. Kraus, "A Stress tensor for Anti-de Sitter gravity," Commun. Math. Phys. 208, 413 (1999), [hep-th/9902121].

[45] S. Hollands, A. Ishibashi and D. Marolf, "Comparison between various notions of conserved charges in asymptotically AdS-spacetimes," Class. Quant. Grav. 22, 2881 (2005), [hep-th/0503045].

[46] I. Papadimitriou and K. Skenderis, "Thermodynamics of asymptotically locally AdS spacetimes," JHEP 0508, 004 (2005), [hep-th/0505190]. 
[47] J. M. Maldacena and A. Strominger, "AdS(3) black holes and a stringy exclusion principle," JHEP 9812 (1998) 005, [hep-th/9804085].

[48] K. Skenderis and S. N. Solodukhin, "Quantum effective action from the AdS / CFT correspondence," Phys. Lett. B 472, 316 (2000), [hep-th/9910023].

[49] D. J. Gross, A. Hashimoto and N. Itzhaki, "Observables of noncommutative gauge theories," Adv. Theor. Math. Phys. 4, 893 (2000), [hep-th/0008075]. 